\title{
Damage constitutive model of stratified cemented backfill based on coupling macroscopic and mesoscopic deformations
}

\author{
Yanan Tang ${ }^{1,2}$, Weidong Song ${ }^{1,2, ~ *}$, Jianxin Fu ${ }^{1,2, *}$ \\ ${ }^{1}$ State Key Laboratory of High-Efficient Mining and Safety of Metal Mines of Ministry of Education, University of Science and \\ Technology Beijing, Beijing 100083, China \\ ${ }^{2}$ School of Civil and Resources Engineering, University of Science and Technology Beijing, Beijing 100083, China
}

\begin{abstract}
The mechanical properties and stress-strain relationship of cemented backfills with different stratified structure have a direct effect on the mining-filling cycle and the mining of adjacent pillars. To obtain the stress-strain evolution curves, the uniaxial compressive strength tests were performed on backfills with stratified numbers of $0,1,2$ and 3 . The deformation of stratified backfill under the compressive load is regarded as a compound of closed deformation of the macroscopic stratified structure and elastic deformation of material. The damage constitutive model of cemented backfills with different stratified structure are established by considering the influence of compacted section. Comparative analysis reveals that the calculated curve based on the established sectional damage constitutive model conforms well to the trial curve. The maximum closed strain of the structural plane has a more significant effect on the mechanical properties of backfill. In the Weibull distribution, with the increase of the parameter $\mathrm{m}$, the peak strength of backfill gradually increases and then reaches to a certain value, and the stress-strain curve gradually becomes steeper, which shows that $\mathrm{m}$ is a reflection of the concentration level of micro-unit strength distribution in the backfill..
\end{abstract}

Backfill mining has significant advantages in reducing tailings and controlling plane subsidence, it also meets the requirements of environmental protection and has become the future development trend of mining ${ }^{[1-4]}$. As we all know, different mines and even different stopes in the same mine have different requirements for the quality of the backfill. The mechanical properties of the backfill are the basic and one of the most important quality evaluation standards for the backfill ${ }^{[5-6]}$.

Currently, there are many studies on the damage constitutive model and strength criterion of the backfill. Literature ${ }^{[7-8]}$ established a constitutive model of uniaxial compression damage of the backfill based on the statistical damage theory. Literature ${ }^{[9]}$ conducted uniaxial compression test with different proportion backfill of a copper mine, and based on the stress-strain curve, the damage evolution equation of the backfill was established. Literature ${ }^{[10]}$ concluded the damage evolution equations of the backfill of different proportions through the uniaxial compression test of the backfill with different proportions. Literature ${ }^{[1]}$ based on the creep test of the backfill and introduced the damage variable into the Burgers model, established the creep statistical damage constitutive model of the backfill. Reference ${ }^{[12]}$ considers the effect of initial temperature on the mechanical properties of the backfill, and combines the theoretical derivation to propose a temperature-time coupling damage constitutive model of the backfill. Literature ${ }^{[13-14]}$ considers factors such as structural size, loose rock weight, surrounding rock force, contact conditions between cemented backfill and surrounding rock, and lateral pressure of tailings backfill on cemented backfill, etc. then established backfill strength model.

The establishment of the above constitutive model and strength criterion are based on the intact backfill, and the influence of the layering effect on the strength and damage evolution of the backfill is not fully considered. Considering only the influence of load on the evolution of the damage of the backfill, there may be some limitations, so it is necessary to contrive a damage constitutive model of the backfill under layering effect and coupling effect of the load.

In this paper, the stress-strain curves of different layered $(0,1,2$, and 3$)$ backfill are obtained through uniaxial compression test. Considering the layered structural plane deformation, micro-unit and microelastic deformation of the cemented backfill, and focusing on the influence of the compacted section of the stress-strain curve, then the damage constitutive models of different layered cemented backfills are established in sections, and finally discuss the influence of each parameter on the results.

\section{Uniaxial compression test of different layered cemented backfill}




\subsection{Test materials}

In the test, the dried tailings and Cementing material of a mine were used to do the laser particle size analysis and the particle size distribution curve of the graded tailings (Fig. 1). The size of the tailings is mainly concentrated in $60 \sim 180 \mu \mathrm{m}$.

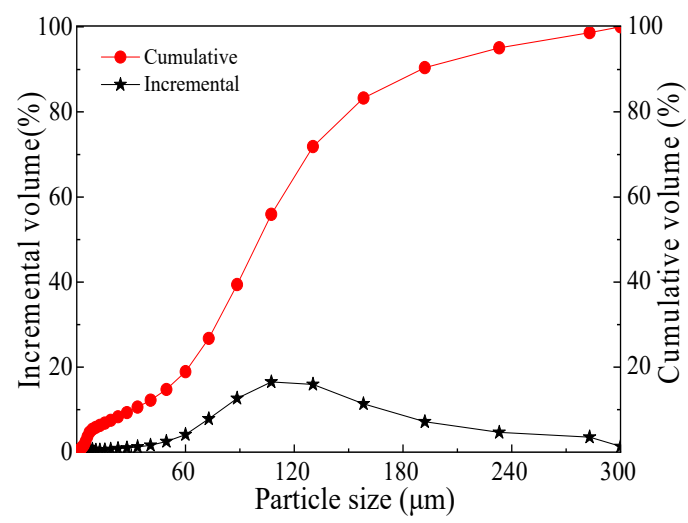

Fig.1 Incremental and cumulative PSD curves of tailings.

\subsection{Make of different layered backfill samples}

The test considers the cemented backfills damage constitutive model of different layers $(0,1,2$ and 3$)$, so making different backfills is critical to the entire test. In this paper, the mold for the backfill is made of transparent acrylic tube with a height of $100 \mathrm{~mm}$ and an outer diameter of $50 \mathrm{~mm}$. The side wall of the transparent tube is affixed with a scale label to facilitate the control of the filling height. Each mold is prepared with two plastic cap which is used to ensure that the backfill is curing into a cylindrical shape. The mold is shown in Fig. 2 (a). 0 layered piece is filled into $100 \mathrm{~mm}$ mold at one time; 1 layered piece is filled into mold in two times, each filling height is $50 \mathrm{~mm}$, and the interval time is $24 \mathrm{~h}$ (depend on the actual production cycle of the mine); similarly, 2 layered piece is filled into mold three times, and the filling height is $33.3 \mathrm{~mm}$ each time (Fig. 2 (b)); 3 layered piece is filled into the mold four times, and the filling height is $25 \mathrm{~mm}$ each time. The cement-sand ratio of all test pieces is set to 1: 6 , and the paste concentration is $70 \%$. The layered backfill pieces are shown in Fig. 2 (c).

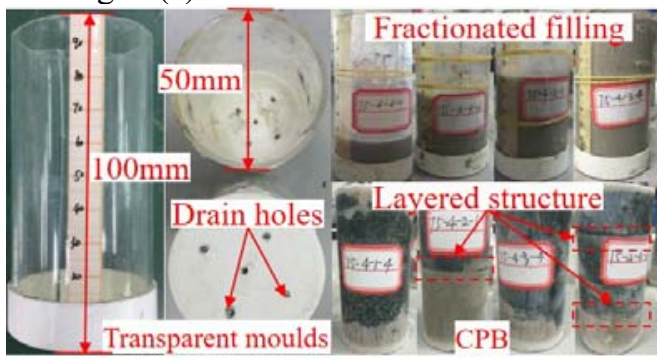

Fig.2 moulds and specimens

\subsection{Backfill strength test}

Place all the finished specimens in a constant temperature $\left(20 \pm 5{ }^{\circ} \mathrm{C}\right)$ and constant humidity $(95 \% \pm$
$5 \%$ ) curing box for curing. In order to minimize the time difference in the making process of the specimens, the standard curing time is 60 days. The GAW-2000 test system was used to test the uniaxial compressive strength of the specimens. Each group of specimens was tested 5 times, and the larger discrete data was eliminated, the remaining data were averaged to obtain the stress-strain curve (see Fig. 3). In view of other scholars' research on cemented backfill, the average elastic modulus E50 is often used to characterize the backfill elastic modulus [12-13], so all the elastic modulus values in this paper are obtained by E50, which is $50 \%$ of the UCS divided by its corresponding strain value.

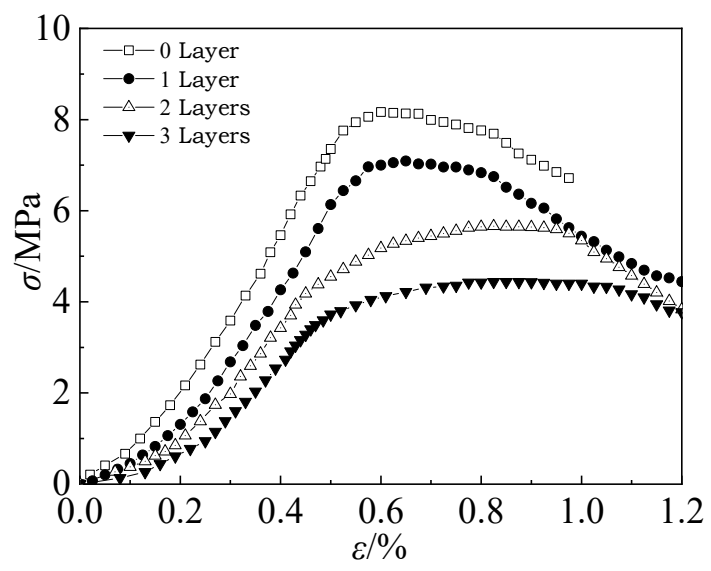

Fig. 3 Stress-strain curves of backfills with different layers

\section{Compressive damage constitutive model}

\subsection{Deformation constitutive equation}

For the intact backfill, although there are no macro defects such as layering on the outside, due to its own consolidation, hydration reaction and other factors, there are a lot of micro pores and micro cracks inside. It is generally believed that the distribution of these microdefects is random, and that the micro-unit strength is subject to the Weibull statistical distribution law [14-15], then the microscopic deformation of the backfill can be expressed as ${ }^{[16]}$ :

$$
\varepsilon_{s}=\frac{\sigma}{E \exp \left[-\frac{1}{m}\left(\frac{\varepsilon}{\varepsilon_{p}}\right)^{m}\right]}
$$

Where $\sigma$ is stress, $\mathrm{MPa} ; \varepsilon$ is strain; $\varepsilon \mathrm{p}$ is the peak strain; $\mathrm{E}$ is the elastic modulus of the intact backfill, GPa; $\mathrm{m}$ is the distribution parameter; $\varepsilon \mathrm{s}$ is the micro-unit strain.

$\mathrm{m}$ can be obtained by the following formula:

$$
m=\frac{1}{\operatorname{In}\left(\frac{E \varepsilon_{p}}{\sigma_{p}}\right)}
$$

where $\sigma \mathrm{p}$ is the peak stress.

For the cemented backfill with macro-layered structural characteristics, under the action of the stress 
$\sigma$, the structural plane is slowly closed, and the calculation formula of the closed strain can be obtained from the literature ${ }^{[17]}$ :

$$
\varepsilon_{j}=\varepsilon_{j m}\left[1-\exp \left(-\frac{\sigma}{E_{j} \varepsilon_{j m}}\right)\right]
$$

where $\varepsilon \mathrm{jm}$ is the maximum closed strain of the layered structural plane; $\varepsilon \mathrm{j}$ is the closed strain of the layered structural plane; $\mathrm{Ej}$ is the closed deformation parameter of the layered structural plane-the closed modulus of the structural plane, MPa.

The closed modulus of the structural plane can be derived by the following method, first obtained by equation (3):

$$
\frac{\varepsilon_{j m}}{\varepsilon_{j m}-\varepsilon_{j}}=\mathrm{e}^{\frac{\sigma}{E_{j} \varepsilon_{j m}}}
$$

Take the logarithm of the two sides of equation (4):

$$
\operatorname{In} \frac{\varepsilon_{j m}}{\varepsilon_{j m}-\varepsilon_{j}}=\frac{\sigma}{E_{j} \varepsilon_{j m}}
$$

The Eq. (5) is organized as follows:

$$
E_{j}=\frac{\sigma}{\varepsilon_{j m} \operatorname{In} \frac{\varepsilon_{j m}}{\varepsilon_{j m}-\varepsilon_{j}}}
$$

\subsection{Compressive deformation constitutive equation}

Under the compressive load, the total deformation of the layered backfill is composed of its internal microscopic deformation and its external macrostructural plane closed deformation, that is, the layered backfill can be regarded as a combination of a intact backfill and a layered structural plane. Idealizing the layered structure plane, it is considered that the mechanical parameters of each layered structure plane are completely the same, from which the compressive deformation damage constitutive equation of the layered backfill is ${ }^{[18]}$ :

$$
\varepsilon=\frac{\sigma}{E \exp \left[-\frac{1}{m}\left(\frac{\varepsilon}{\varepsilon_{p}}\right)^{m}\right]}+n \varepsilon_{j m}\left[1-\exp \left(-\frac{\sigma}{E_{j} \varepsilon_{j m}}\right)\right]
$$

Where $\mathrm{n}$ is the number of layers.

\subsection{Modification of constitutive equation}

By analyzing the constitutive model obtained by Eq. (7), when the stress is low, it is found that the stress-strain curve obtained by fitting is approximately upward convex. However, the experiment results show that when the stress is lo, there is an obvious compaction stage, and the curve becomes a concave shape. If the constitutive model of Eq. (7) is used, the compaction stage of the backfill cannot be accurately characterized.

The literature ${ }^{[19-20]}$ explained in detail that there is an obvious compaction stage of the backfill under uniaxial compression strength experiment, and the evolution law of the stress-strain curve is basically the same, and the curves are all concave, so in the study of the constitutive model, the stage of stress-strain curve compaction should be focused on.

As can be seen from the stress-strain curve of the backfill in Fig. 4, the uniaxial compressive stress-strain curve of the backfill is concave, and the slope of the curve tends to increase with the increase of the loading stress, showing obvious nonlinear characteristics.

In the analysis of the compression damage constitutive model of the backfill, the compaction stage of stress-strain curve should be considered separately, assuming that the stress and strain at the end of the compaction stage are $\sigma \mathrm{f} 、 \varepsilon f$ respectively. Literature [14] believes that the shape of the stress-strain curve is mainly controlled by the Eq. (7), according to the stressstrain curve with characteristics of concave during the compaction stage, so changing this part to, then obtained the constitutive equation, shown in Eq. (8). After the compaction stage, the constitutive equation is still expressed by Eq. (7), then the uniaxial compression constitutive model of different layered cemented backfill can be expressed as:

$$
\left\{\begin{array}{c}
\varepsilon=\frac{\sigma}{E\left\{1-\exp \left[-\frac{1}{m}\left(\frac{\varepsilon}{\varepsilon_{p}}\right)^{m}\right]\right\}}+n \varepsilon_{j m}\left[1-\exp \left(-\frac{\sigma}{E_{j} \varepsilon_{j m}}\right)\right] \quad \varepsilon \leq \varepsilon_{f} \\
\varepsilon=\frac{\sigma}{E \exp \left[-\frac{1}{m}\left(\frac{\varepsilon}{\varepsilon_{p}}\right)^{m}\right]}+n \varepsilon_{j m}\left[1-\exp \left(-\frac{\sigma}{E_{j} \varepsilon_{j m}}\right)\right] \quad \varepsilon>\varepsilon_{f}
\end{array}\right.
$$

\subsection{Determination of constitutive equation parameters}

According to the stress-strain curve of the backfill, the elastic modulus $\mathrm{E}$ (the elastic modulus of the intact backfill), the end stress $\sigma \mathrm{f}$ and strain $\varepsilon \mathrm{f}$ of the compaction stage, the peak stress $\sigma$ p, the peak strain $\varepsilon$ $\mathrm{p}$, and the distribution parameter $\mathrm{m}$; According to the strain difference relationship between one structural plane backfill under the stress $\sigma \mathrm{x}(\sigma \mathrm{x}>\sigma \mathrm{f})$ and the intact backfill under the same stress, the maximum closed strain $\varepsilon$ jm of the structural plane can be obtained, and then substitute it into Eq. (6), Combining the stressstrain curve, the closed plane elastic modulus Ej can be obtained. Through the above calculation and derivation, the parameters of the constitutive equations of different layered cemented backfill can be obtained, as shown in Table 1.

By regression analysis of the parameters of the damage constitutive model of different layered cemented backfills in Table 1, a unified damage constitutive model considering the number of layered structures was established. The end stress and strain, peak stress and peak strain of the cemented backfill during the compaction stage can be quantitatively characterized by the number of layers $\mathrm{n}$, and the quantitative relationship is as Eq. (9) to (12).

$$
\begin{gathered}
\sigma f=2.59-0.51 \mathrm{n}+0.07 \mathrm{n} 2, \quad \mathrm{R} 2=0.99 \\
\varepsilon \mathrm{f}=0.12+0.03 \mathrm{n}, \quad \mathrm{R} 2=0.98
\end{gathered}
$$




$$
\begin{gathered}
\sigma p=8.23-1.26 n, \quad R 2=0.98 \\
\varepsilon p=0.443+0.14 \exp (0.28 n), \quad R 2=0.97
\end{gathered}
$$

Substitute Eqs. (11) and (12) into Eq. (2) to get the expression of parameter $\mathrm{m}$ :

$$
m=\frac{1}{\operatorname{In}\left[\frac{8.18+2.6 \exp (0.28 n)}{8.23-1.26 n}\right]}
$$

Then the damage constitutive model of cemented backfill can be expressed as:

$$
\begin{cases}\varepsilon=\frac{0.054 \sigma}{1-\exp \left\{-\frac{1}{m}\left[\frac{\varepsilon}{0.443+0.14 \exp (0.28 n)}\right]^{m}\right\}}+0.018 n[1-\exp (-10.7 \sigma)] & \varepsilon \leq \varepsilon_{f} \\ \varepsilon=\frac{0.054 \sigma}{\exp \left\{-\frac{1}{m}\left[\frac{\varepsilon}{0.44+0.14 \exp (0.28 n)}\right]^{m}\right\}}+0.018 n[1-\exp (-10.7 \sigma)] & \varepsilon>\varepsilon_{f}\end{cases}
$$

Combining Eqs. (13) and (14), the damage constitutive equations of different layered cemented backfill can be obtained as follows:

It should be noted that the specific parameters in Eqs. (15) to (18) may be different due to the difference of cement-sand ratio, paste concentration, curing time and aggregate composition, but this damage constitutive equation can be used as a reference for other cemented backfill.

\section{Verification and the influence of various parameters on the results}

\subsection{Damage constitutive model verification}

According to Eqs. (15) to (18), the uniaxial compressive stress-strain curve of different layered cemented backfill can be calculated and compared with the test curve (Fig. $4)$.
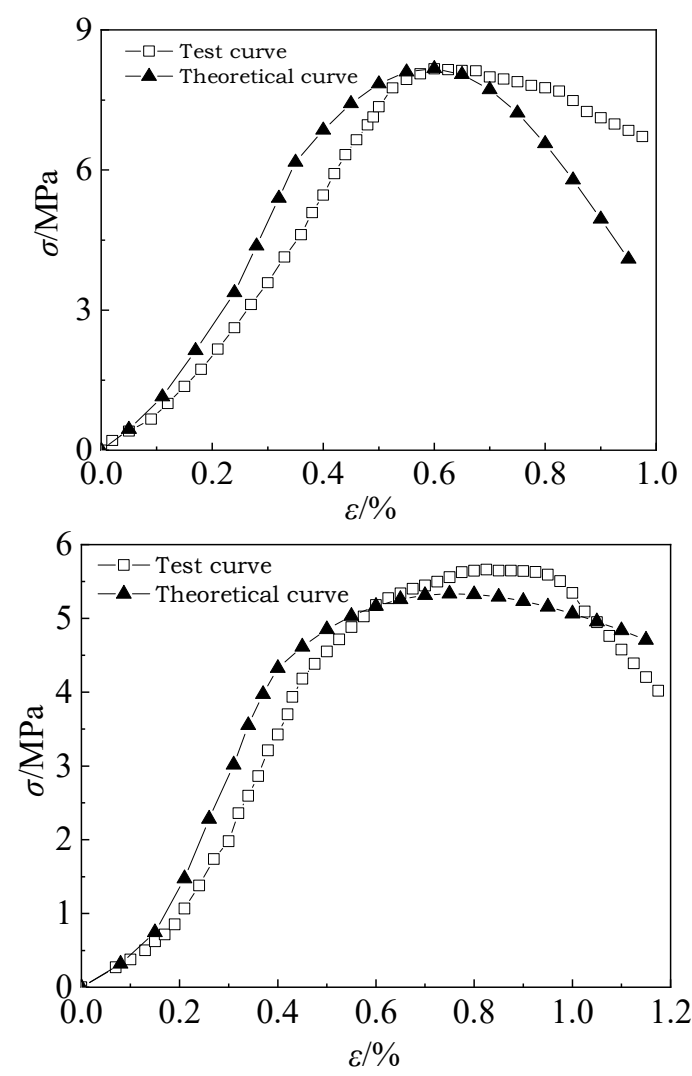

Fig.4 Calculated and experimental stress-strain curves of uniaxial compression: (a) 0 Layer; (b) 2 Layers.

It can be seen from table 2 and Fig. 5:

(1) The uniaxial compression stress-strain curves of different layered cemented backfill all have obvious compaction stages. On the one hand, during the preparation of the backfill, due to the unevenness of the aggregate, a large number of micro porosity and microfissures were formed; on the other hand, in the process of constructing the damage constitutive model of the backfill, the nonlinear characteristics of the compaction stage must be considered separately.

(2) The peak strength of different layered cemented backfill is different, and its uniaxial compressive strength decreases as the number of layers increases. When the number of layers increases from 0 to 2 , the uniaxial compressive strength decreases by $30.7 \%$, indicating that the layered structure has a degrading effect on the strength of the backfill.

(3) The calculation results of the damage constitutive model established in consideration of the impact of the compaction phase are in good agreement with the testing curves from the initial loading to the peak phase, indicating that the damage constitutive model established considering the impact of the compaction phase is reasonable and feasible.

\subsection{The effect of the parameter $\mathrm{m}$ on the mechanical properties of the backfill}

In order to study the influence of the parameter $m$ on the mechanical properties of the backfill, keep E $=1.86 \mathrm{GPa}$, $\varepsilon j \mathrm{~m}=0.018, \mathrm{Ej}=518 \mathrm{MPa}$ unchanged, change the value 
of $\mathrm{m}$, the model calculates the stress-strain curve and the peak strength and peak strain change with $\mathrm{m}$. The curve is shown in Fig. 5.
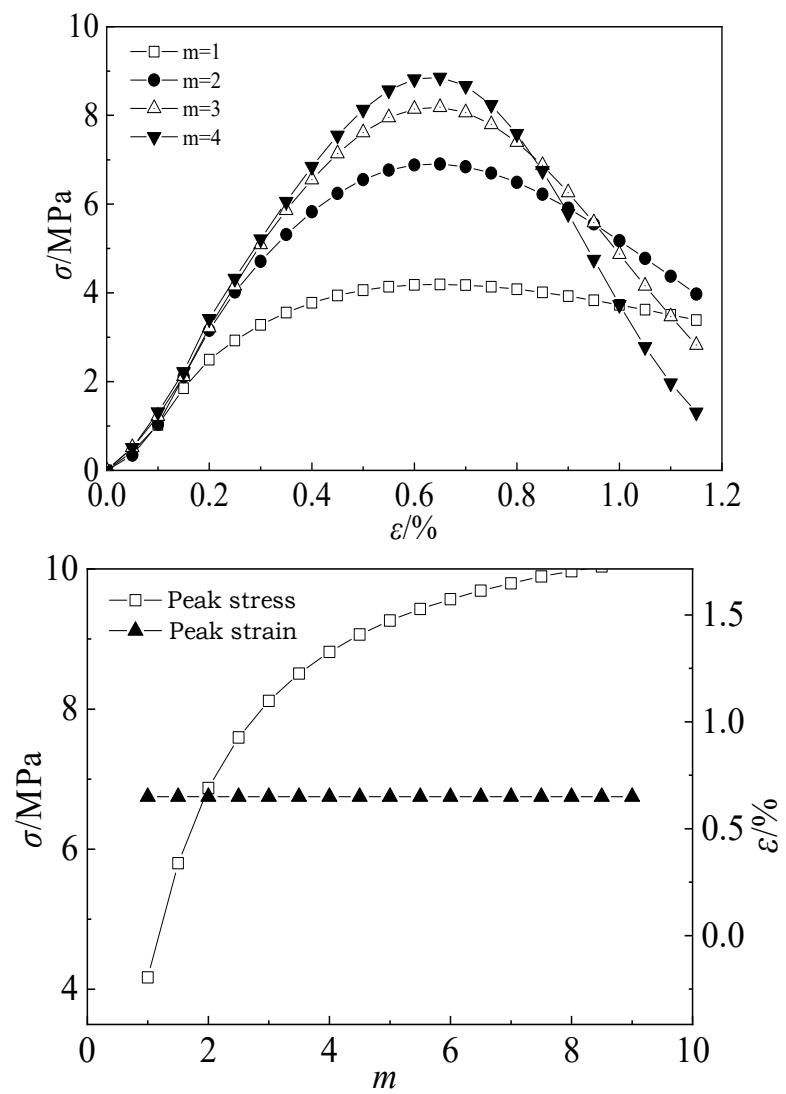

Fig.5 Effect of m on sample's mechanical property: (a) stressstrain curves; (b) peak stress and strain.

As can be seen from Fig.5, with the increase of the parameter $\mathrm{m}$, the stress-strain curve of the specimen gradually slows down, the material unit-strength distribution range gradually widens, and the peak strength continues to increase. When $\mathrm{m}$ increases to a certain degree, the peak strength of the specimen tends to be a fixed value, and its influence on the mechanical properties of the backfill gradually decreases, no matter how $m$ changes, it has little effect on the peak strain of the test piece. Based on the above analysis, it is believed that the parameter $m$ is a reflection of the concentration of unit-strength distribution within the backfill.

\section{Conclusion}

(1) With the help of structural mechanics theory, the deformation of the cemented backfill is regarded as the superposition of the closed deformation of the macroscopic layered structural plane of the backfill and the microscopic mesoscopic elastic deformation. Mainly considering the effect of the compaction stage and the damage constitutive model of different layered cemented backfill are established in stages.

(2) Comparative analysis shows that the calculated curve of the segmented damage constitutive model is in good agreement with the testing curve, indicating that it can better characterize the damage deterioration effect of different layered cemented backfill under uniaxial compression.

(3) The strength of the backfill decreases with the increase of the layered structure plane, which is reflected by the weakening effect of the structure on the strength of the backfill. Compared with the closed modulus of the structural plane, the maximum closed strain of the structural plane has a more significant effect on the mechanical properties of the backfill. In the Weibull distribution, with the increase of the parameter $m$, the peak strength of the backfill gradually increases and then tends to a certain value, and the stress-strain curve gradually becomes steeper. It is considered that the parameter $\mathrm{m}$ is a reflection of the concentration of the unit-strength distribution inside the backfill.

\section{References}

1. SONG Weidong, CAO Shuai, FU Jianxin, et al. Sensitivity analysis of impact factors of pillar stability and its application[J]. Rock and Soil Mechanics, 2014, 35(sup. 1): 271-277.

2. CAO Shuai, SONG Weidong, DENG Dan, et al. Numerical simulation of land subsidence and verification of its character for an iron mine using sublevel caving $[\mathrm{J}]$. International Journal of Mining Science and Technology, 2016, 26(2): 327-332.

3. LI Li, MICHEL A. An improve method to assess the required strength of cemented backfill in underground stopes with an open face[J]. Journal of China University of Mining \& Technology, 2014, 24(4): 549-558.

4. JU Feng, LI Meng, ZHANG Jixiong, et al. Construction and stability of an extra-large section chamber in solid backfill mining[J]. Journal of China University of Mining \& Technology, 2014(6): 763-768.

5. WU Aixiang, WANG Yong, WANG Hongjiang. Estimation model for yield stress of fresh uncemented thickened tailings: coupled effects of true solid density, bulk density, and solid concentration. International Journal Miner Process, 2015, 143: 117-124.

6. WU Aixiang, WANG Yong, WANG Hongjiang, et al. Coupled effects of cement type and water quality on the properties of cemented paste backfill[J]. International Journal Miner Process, 2015, 143: 6571.

7. ZHAO Shuguo, SU Dongliang, WU Wenrui, et al. Study on damage model of backfill based on Weibull distribution under uniaxial compression[J]. China Mining Magazine, 2017, 26(2): 106-111.

8. LIU Zhixiang, LIU Qingling, DANG Wendang. On softening-hardening intrinsically constitutive model for damage of tailings-cemented backfill[J]. Journal of Shandong University of Science and Technology (Natural Science), 2012, 31(2): 36-41.

9. DENG Daiqiang, YAO Zhongliang, TANG Shaohui, et al. Study on constitutive model of damage of 
backfill under uniaxial compressive loading[J]. Soil Engineering and Foundation, 2006, 20(3): 53-55.

10. ZHANG Fawen. Investigations on Solidified Characteristics and Mechanism of Slag Cementitious Materials in Mine Filling[D]. Wuhan: Wuhan University, 2009.

11. ZHAO Shuguo, SU Dongliang, ZHANG Yalun, et al. Study on Creep Test of cemented Tailings Backfill and Statistical Damage Model[J]. Metal Mine, 2016, (5): 26-30.

12. WANG Yong, WU Aixiang, WANG Hongjiang, et al. Damage constitutive model of cemented tailing paste under initial temperature effect[J]. Chinese Journal of Engineering, 2017, 39(1): 31-38.

13. CAO Shuai, SONG Weidong, XUE Gaili, et al. Mechanical characteristics variation of stratified cemented tailing backfilling and its failure modes[J]. Journal of China University of Mining \$ Technology, 2016, 45(4): 717-722+728.

14. DENG Huafeng, HU Anlong, LI Jianlin, et al. Statistical damage constitutive model of sandstone under water-rock interaction[J]. Rock and Soil Mechanics, 2017, 38(3): 631-639.
15. YUAN Xiaoqing, LIU Hongyan, LIU Jingping. Constitutive model of rock mass with non-persistent joints based on coupling macroscopic and mesoscopic damages[J]. Rock and Soil Mechanics, 2015, 36(10): 2804-2814.

16. LIU Hongyan, HUANG Yushi, LÜ Shuran, et al. A compression damage constitutive model of jointed rock mass based on deformation components[J]. Journal of Central South University (Science and Technology Edition), 2014, 45(6): 2014-2020.

17. SUN Guangzhong. Structural mechanics of rock mass[M]. Beijing: Science Press, 1988: 124-127.

18. SUN Guangzhong, SUN Yi. Principle of rock mass mechanics[M]. Beijing: Science Press, 2011: 44-50.

19. CAO Shuai, SONG Weidong, XUEGaili, et al. Tests of strength reduction of cemented tailings filling considering layering character.

20. FU Jianxin, DU Cuifeng, SONG Weidong. Strength sensitivity and failure mechanism of full tailings cemented backfills. 